116. Herr Kessler, Gustav, Baumeister u. Civil-Ingenieur, Inse] Hiddens-Oee u. Stralsund.

117. "Naumann, Alexander, Kaufmann, in Zittan.

118. " Becher, Hermann, Particulier, in Süderstape, Schleswig.

\title{
1871 .
}

119. Herr Breh m, Reinhold, Doctor, Arzt der Gesandtschaften zu Madrid.

120. "Klippert, Gust. Herm., Kaufmann, in Vegesack bei Bremeri.

121. " von Löwis, O., zu Kudling, Livland.

122. " von der Gabelentz, Grossherzoglich Sächsischer Kammerherr, in Münchenbernsdorf, Sachsen-Weimar.

123. Mr. Sharpe, R. B., Bibliothekar der Zoologischen Gesellschaft zu London.

124. Herr Born, Emil, Hauptmann im eidg. Generalstab, in Herzogenbuchsee, Schweiz.

125. " Girtanner, A., Doctor medicinae, in St. Gallen, Schweiz.

126. ” Friele, Hermann, in Bergen, Norwegen.

127. Mr. Sclater, Ph. L., Doctor, Secretär der Zoologischen Gesellschaft zu London.

128. Herr Stejueger, Student, in Bergen, Norwegen.

Ferner hinzutretende Mitglieder werden demnächst im Journale verzeichnet werden. Um etwaige Veränderungen oder Berichtigungen der Adressen wird ergebenst gebeten.

Berlin, im Januar 1871.

J. Cabanis, Secr.

\section{Benachrichtigung an die Mitglieder.}

Diejenigen Mitglieder, welche in Folge der früheren Ben a chrichtigung (September-Heft 1870, S. 399 u. 400) den Jahresbeitrag pro 1871 zur Gesellschaftskasse bereits eingesandt haben, finden die Mitgliedskarte für das laufende Jahr, welche zugleich als Quittung dient, hier beigefügt. Restanten mögen hiermit, statt besonderer Zuschrift, an die Einsendung (thunlichst durch PostAnweisung) des nuumehr fälligen Beitrages erinnert sein.

Dr. J. Cabanis, Secr.

(Berlin, Simeons-Str. 23.) 